\title{
On the use of a temperature based friction model for a virtual force sensor in industrial robot manipulators
}

\author{
Luca Simoni $^{1}$, Enrico Villagrossi ${ }^{2}$, Manuel Beschi ${ }^{2}$, Alberto Marini ${ }^{1}$, \\ Nicola Pedrocchi ${ }^{2}$, Lorenzo Molinari Tosatti ${ }^{2}$, Giovanni Legnani ${ }^{1}$, Antonio Visioli ${ }^{1}$ \\ ${ }^{1}$ Department of Mechanical and Industrial Engineering, University of Brescia, Brescia, Italy \\ ${ }^{2}$ Institute of Industrial Technologies and Automation (ITIA-CNR), National Research Council, Milan, Italy
}

This is the pre-peer reviewed version of the following article: On the use of a temperature based friction model for a virtual force sensor in industrial robot manipulators, which has been published in final form at 10.1109/ETFA.2017.8247655. This article may be used for non-commercial purposes in accordance with Journal terms and conditions for Self-Archiving.

\begin{abstract}
In this paper we propose the use of a dynamic model in which the effects of temperature on friction are considered to develop a virtual force sensor for industrial robot manipulators. The estimation of the inertial parameters and of the friction model are explained. The effectiveness of the virtual force sensor has been proven in a polishing task. In fact, the interaction forces between the robot and the environment has been measured both with the virtual force sensor and a common load cell. Moreover, the advantages provided by considering the temperature dependency are highlighted.
\end{abstract}

\section{INTRODUCTION}

It is nowadays clear that in order to improve the performance of industrial robots, or to increase the number of working applications of standard industrial manipulators, it is necessary to have a complete and reliable dynamic model of the robot. Several methods related to the dynamic model identification have been presented in the literature (see, for example, [1], [2]). The majority of them agrees that the accuracy of the dynamic model can be improved by using a proper friction model [3]. Several friction models presented in the literature are nowadays adopted in mechatronics (see for example [4], [5], [6], [7] or [8]), and recently, the dependency of friction force on the temperature have been more and more analysed [9], [10], [11], [12]. A complete dynamic model of a robot manipulator can be used for several applications in a large variety of working areas. Examples of these applications can be: machining tasks performed with robots (milling, deburring or polishing); the minimization of the energy consumption during the manipulator motion like in pick and place applications [13]; the maintenance time prediction of the mechanical parts (like motors or speed reducers, or the lubricant substitution time prediction); the implementation of a virtual force sensor for different applications and to ensure the safety of the robot cell; the accomplishment of cooperative tasks between robots and humans. In this work, the purpose of the use of a complete dynamic model of a robot manipulator is the implementation of a virtual force sensor. It is worth stressing that, if the model is sufficiently good, the use of a virtual force sensor, instead of the use of a force/torque sensor, can reduce the application costs up to $€ 5000$. Different approaches for the implementation of a virtual force sensor are reported in the literature. A first common approach can be found in [14] or [15], where the end-effector force wrench is computed by subtracting the torques estimated with the dynamic model from the measured torques. Another approach is presented in [16] and extended in [17] where a disturbance observer is used to avoid the problems related to noisy accelerations. This work follows the approach proposed in [14] and [15] and exploits the use of a thermal based friction model to improve the accuracy of the dynamic model of the robot. In particular, an extended version of the thermal friction model proposed and analysed in [9] and [10] has been used. The extension consists in adding the Stribeck effect to the thermal model. The developed virtual force sensor has been used in a polishing task to measure the interaction force between the robot and the evironment. To validate the virtual force sensor, the value of the force estimated have been compared with the force measured with a real load cell.

This paper is structured as follows: the considered friction model is presented in Section II, the experimental setup is described in Section III and the identification procedure for both the inertial model and the friction one is presented in Section IV. The proposed virtual force sensor is explained in Section V, the test protocol is presented in Section VI and the results of the work are presented in Section VII. Finally, concluding remarks and future works are in Section VIII. 


\section{CONSIDERED FRICTION MODEL}

The base function for the friction model that has been used in this work is composed by a third order polynomial function $\tau_{f p o l}$ (as in [9], [10])

$$
\tau_{f p o l}=\left[c_{0}+c_{1}|\dot{q}|+c_{2}|\dot{q}|^{2}+c_{3}|\dot{q}|^{3}\right] \operatorname{sgn}(\dot{q})
$$

plus a Stribeck exponential term $\tau_{f s t r}$ (see, for example, [4])

$$
\tau_{f s t r}=c_{4} e^{-h|\dot{q}|} \operatorname{sgn}(\dot{q})
$$

So, at each time instant and for all the robot joints, the relation between the friction torque and the joint speed is represented as

$$
\tau_{f}=\left[c_{0}+c_{1}|\dot{q}|+c_{2}|\dot{q}|^{2}+c_{3}|\dot{q}|^{3}+c_{4} e^{-h|\dot{q}|}\right] \operatorname{sgn}(\dot{q}),
$$

where $\dot{q}$ is the single motor speed, $c_{0}, c_{1}, c_{2}$ and $c_{3}$ are the coefficients of the polynomial function $\tau_{f p o l}$, and $c_{4}$ and $h$ are the coefficients of the Stribeck function and $\operatorname{sgn}(\dot{q})$ represents the sign function applied to the measured motor speed $\dot{q}$. As in [9], the coefficients $c_{0} \ldots c_{4}$ have been considered to be dependent on the joint internal temperature. In particular, both polynomial $\left(c_{0} \ldots c_{3}\right)$ and Stribeck $\left(c_{4}\right)$ coefficients have been supposed to be linearly dependent on the temperature. However, due to the fact that they are related to two different friction phenomena, the two dependencies have been kept separated. For the polynomial coefficients, the temperature dependency has been expressed as

$$
c_{i}(T)=c_{i}\left(T_{0}\right)\left[\alpha\left(T-T_{0}\right)+\beta\right] \quad \text { for } i=1 \ldots 3
$$

while the one related to the Stribeck term has been expressed as

$$
c_{4}(T)=c_{4}\left(T_{0}\right)\left[\gamma\left(T-T_{0}\right)+\delta\right],
$$

where $T_{0}$ is the value of the joint internal temperature during the experiment used to identify the friction parameters $c_{1} \ldots c_{4}$ (see Section IV). As it is possible to notice, the exponential parameter $h$ has been kept constant for the sake of simplicity. It is worth stressing that, in case of perfect modelling the values of $\beta$ and $\delta$ are equal to 1 ; however, different values of these two parameters are expected because of the unavoidable presence of noise and modelling errors in the identification phase. The dependency from the temperature of the polynomial term and the Stribeck term have been kept separated because, as shown in [18], there are different behaviours of the system at low and high velocities (where the Stribeck and the polynomial terms are more relevant, respectively). In particular, it is worth highlighting the following considerations:

- if the temperature increases, the lubricant viscosity decreases, therefore,

- if the velocity is low the lubricant efficiency to separate the surfaces in contact decreases and, thus, the friction torque (that is mainly due to the Stribeck term because the velocity is low) increases;

- if the velocity is high the lubricant efficiency to resist to the body in motion decreases and, thus, the friction torque decreases.

- if the temperature decreases, the lubricant viscosity increases, therefore,

- if the velocity is low the lubricant efficiency to separate the surfaces in contact increases and, thus, the friction torque (that is mainly due to the Stribeck term because the velocity is low) decreases;

- if the velocity is high the lubricant efficiency to resist to the body in motion increases and, thus, the friction torque increases.

By taking into account these considerations, the complete thermal friction model can be written as

$$
\begin{aligned}
\tau_{f}(T)= & \tau_{f 0 p o l}\left[\alpha\left(T-T_{0}\right)+\beta\right]+\tau_{f 0 s t r}\left[\gamma\left(T-T_{0}\right)+\delta\right]= \\
& =\left(c_{0}+c_{1}|\dot{q}|+c_{2}|\dot{q}|^{2}+c_{3}|\dot{q}|^{3}\right) \operatorname{sgn}(\dot{q})\left[\alpha\left(T-T_{0}\right)+\beta\right] \\
& +c_{4} e^{-h|\dot{q}|} \operatorname{sgn}(\dot{q})\left[\gamma\left(T-T_{0}\right)+\delta\right],
\end{aligned}
$$

where the temperature $T$ can be computed by the first-order differential equation

$$
\frac{d T}{d t}=\left[\dot{q}\left(\tau_{f p o l}+\tau_{f s t r}\right)-K\left(T-T_{\text {env }}\right)\right] \frac{1}{C},
$$

which is a simple equation of heat exchange between bodies and air, where $C$ is the equivalent thermal capacity of the joint, $K$ is the coefficient of the dissipated power, $\dot{q}$ is the joint velocity and $T_{e n v}$ is the environmental temperature.

As it is possible to notice from [9], the proposed friction model automatically updates the joint internal temperature estimates. This operation is done as follows: at each sampling time $k$, the new value of the joint internal temperature is computed by means of

$$
T_{k}=T_{k-1}+\left[\dot{q}_{k-1}\left(\tau_{f p o l_{k-1}}+\tau_{f s t r_{k-1}}\right)-K\left(T_{k-1}-T_{e n v}\right)\right] \frac{1}{C} t_{s}
$$


where $t_{s}$ is the sampling period. Subsequently, the value of the friction torque is computed by using

$$
\tau_{f_{k}}=\tau_{f 0 p o l_{k}}\left[\alpha\left(T_{k}-T_{0}\right)+\beta\right]+\tau_{f 0 s t r_{k}}\left[\gamma\left(T_{k}-T_{0}\right)+\delta\right] .
$$

It is worth stressing that $T_{e n v}$ is the only measurement needed for the use of the model.

As the joint initial internal temperature is unknown at the beginning of the robot work, an observer based strategy has been adopted to estimate its value. The observer has been necessary only at the beginning of the process and its only purpose has been to detect the initial values of the joints internal temperatures. The observer law has been expressed, for each joint, as

$$
\begin{aligned}
T_{k}= & T_{k-1}+\left[\dot{q}_{k-1}\left(\tau_{f p o l_{k-1}}+\tau_{f s t t_{k-1}}\right)-K\left(T_{k-1}-T_{e n v}\right)\right] \frac{1}{C} t_{s} \\
& -G\left(\tau_{R M \text { Smeas }_{k-1}}-\tau_{R M S_{k-1}}\right) t_{S}
\end{aligned}
$$

where $T_{k}$ is the estimated temperature value at time instant $k, T_{k-1}$ is the estimated temperature value at the previous time instant $(k-1), \dot{q}_{k-1}$ is the measured motor speed at previous time instant, $\tau_{R M \text { Smeas }_{k-1}}$ is the RMS value of the measured friction torque for the previous $N$ samples at previous time instant (usually $N=20$ ), $\tau_{R M S_{k-1}}$ is the RMS value of the estimated friction torque for the previous $N$ samples at previous time instant and $G$ is the observer gain.

It is worth stressing that: due to the fact that both the polynomial friction torque value $\left(\tau_{f p o l}\right)$ and the Stribeck one $\left(\tau_{f s t r}\right)$ have been computed by considering the sign of the motor speed $(\operatorname{sgn}(\dot{q}))$, the product between the motor speed $\dot{q}$ and the sum of the two different friction torques is always positive, and it represent the injected thermal power to the robot joint. It is worth noting that, in order to avoid the discontinuity of the friction torque model at zero velocity, the sign function has been substituted with a linear function (in order to keep the continuity of the friction function) at (very low) velocities below a given threshold $\dot{q}_{t h}$, so that

$$
\tau_{f}=\left\{\begin{array}{r}
{\left[c_{0}+c_{1}|\dot{q}|+c_{2}|\dot{q}|^{2}+c_{3}|\dot{q}|^{3}+c_{4} e^{-h|\dot{q}|]} \operatorname{sgn}(\dot{q})\right.} \\
\text { if abs }(\dot{\mathrm{q}}) \geq \dot{\mathrm{q}}_{\text {th }} \\
{\left[c_{0}+c_{1} \dot{q}_{t h}+c_{2} \dot{q}_{t h}^{2}+c_{3} \dot{q}_{t h}^{3}+c_{4} e^{-h \dot{q}_{t h}}\right] \frac{\dot{q}}{\dot{q}_{t h}}} \\
\text { if abs }(\dot{\mathrm{q}})<\dot{\mathrm{q}}_{\text {th }}
\end{array}\right.
$$

\section{EXPERIMENTAL SETUP}

The experimental setup available in this work consists of a standard industrial Comau NS16 1.65 Hand manipulator. It is a six degrees of freedom robot with an anthropomorphic open chain (see Figure 1) with a maximum payload of $16 \mathrm{~kg}$. The joints are actuated by six AC brushless LAFERT servomotors. Different types of speed reducers are mounted on the different joints (see Table I). The robot is equipped with a C5GOpen controller and interfaced with ROS control node

\begin{tabular}{|c|c|c|c|}
\hline Axis & Reduction rate & Type & Lubrication \\
\hline 1 & -181.5873 & Gears & oil \\
\hline 2 & 159.2609 & Cycloidal & oil \\
\hline 3 & 169.4737 & Cycloidal & oil \\
\hline 4 & 72.5860 & Gears & oil \\
\hline 5 & 78.7500 & Harmonic Drive & grease \\
\hline 6 & -50.0000 & Harmonic Drive & grease \\
\hline
\end{tabular}

TABLE I

REDUCTION RATES OF THE SPEED REDUCERS FOR EACH JOINT.

(http://www.ros.org/).

A pneumatic Schunk gripper is attached to the robot end-effector. The fingers of the gripper are designed to handle the cast iron sample to polish. The fingers of the gripper have been instrumented with two Phidgets load cells in order to measure the interaction force between the robot and the environment (Figure 2) and to compare it with the one estimated with the proposed virtual force sensor. The load cells are single axis sensors, therefore they have been mounted in order to measure the force along the direction required for the polishing task (i.e., the direction perpendicular to the disc sander surface). In order to polish the iron sample, a standard rotating disc sander has been used. 


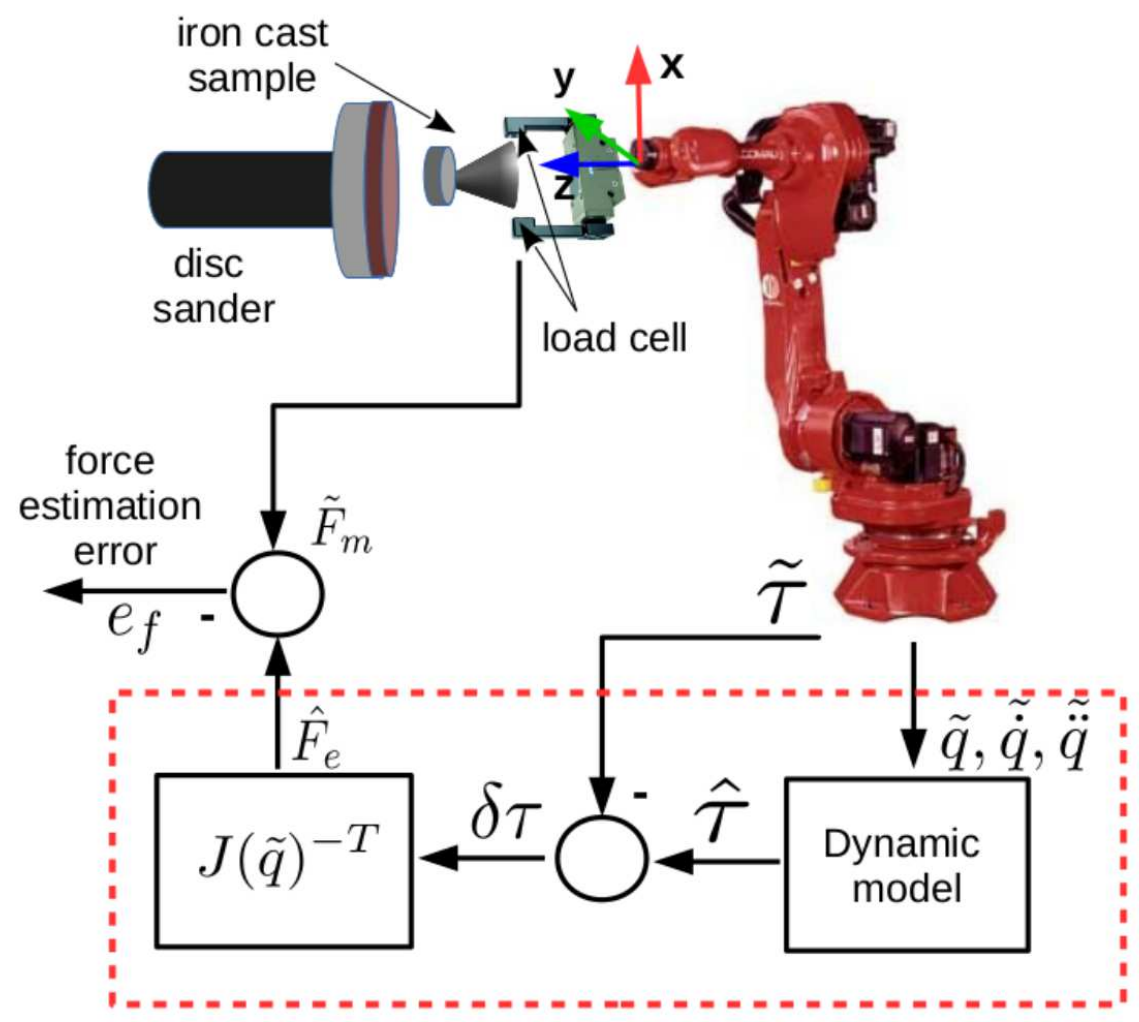

Virtual Force Sensor

Fig. 1. The experimental setup.

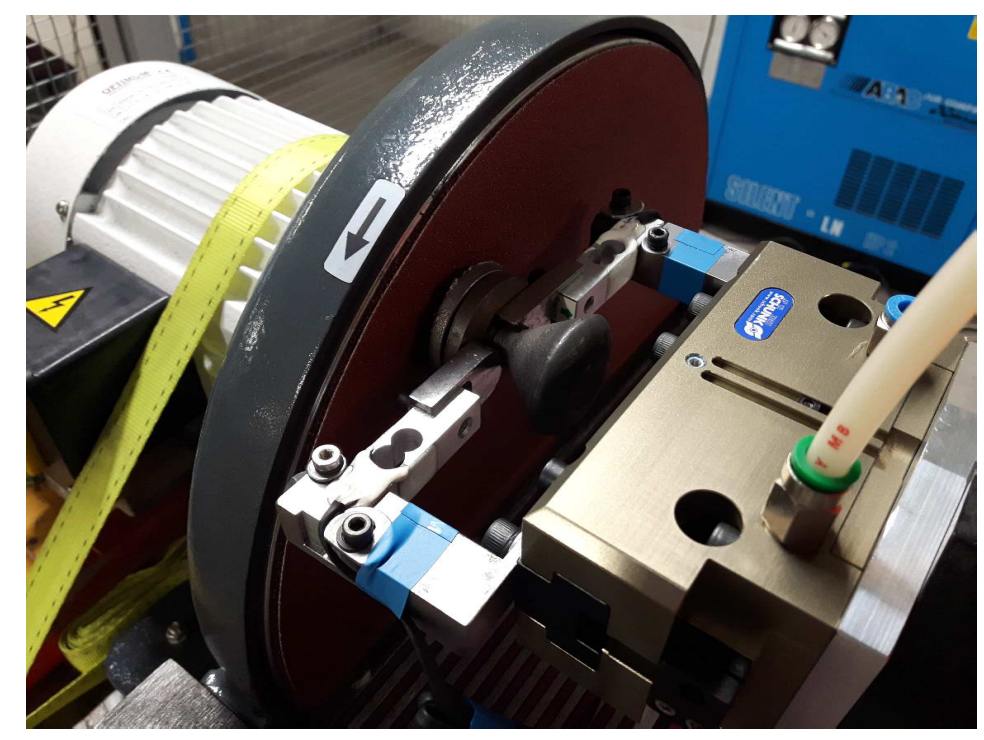

Fig. 2. Robot end effector in contact with the sander disk.

\section{IDENTIFICATION EXPERIMENTS}

The complete dynamic model identification of the open chain rigid robot is based on the well-known dynamic equation [19]

$$
\boldsymbol{\tau}=\mathbf{B}(\mathbf{Q}) \ddot{\mathbf{Q}}+\mathbf{C}(\mathbf{Q}, \dot{\mathbf{Q}}) \dot{\mathbf{Q}}+\mathbf{G}(\mathbf{Q})+\tau_{f}
$$


where $\mathbf{Q}, \dot{\mathbf{Q}}$ and $\ddot{\mathbf{Q}}$ are the vectors of the joints positions, velocities and accelerations, $\tau$ is the vector of the joint torques, $\mathbf{B}(\mathbf{Q})$ is the inertia matrix, $\mathbf{C}(\mathbf{Q}, \dot{\mathbf{Q}})$ is the Coriolis matrix, $\mathbf{G}(\mathbf{Q})$ is the gravitational term and $\tau_{f}$ is the friction term. This equation can be rewritten in a more compact form as

$$
\tau=\overline{\boldsymbol{\Phi}} \mathbf{X}
$$

where $\mathbf{X}$ represents the parameters vector to estimate, $\boldsymbol{\tau}$ is the vector of the measured motors torques and $\overline{\boldsymbol{\Phi}}$ is the regression matrix as explained in [2] and [20].

The estimation experiments have been performed for both inertial and friction parameters.

First of all, in order to identify the inertial and friction parameters at a certain environmental temperature $T_{e n v}=T_{0}$, a local based identification approach has been used. In fact, as demonstrated in [20], a task oriented local calibration can be a suitable choice to improve the model prediction accuracy. Due to this consideration, a proper local exciting trajectory has been designed. After the experimental session, the data are collected in a compound regression matrix $\overline{\mathbf{\Phi}}$ and the inertial and the friction parameters at temperature $T_{0}$ can be computed as (see [20])

$$
\mathbf{X}=\overline{\boldsymbol{\Phi}}^{+} \tau
$$

in which $\overline{\boldsymbol{\Phi}}^{+}$is the Moore-Penrose pseudo-inverse of matrix $\overline{\boldsymbol{\Phi}}, \mathbf{X}$ represents the estimated parameters vector and $\tau$ is the vector of the measured motors torques at each sampling time. The parameters vector $\mathbf{X}$ has been expressed as

$$
\mathbf{X}=\left[\begin{array}{ll}
\mathbf{X}_{J} & \mathbf{X}_{f}
\end{array}\right]^{T}
$$

in which $\mathbf{X}_{J}$ is the vector of the inertial parameters and $\mathbf{X}_{f}$ is a vector that contains all the friction parameters $c_{1} \ldots c_{4}$ for all the six robot joints at temperature $T_{0}$. In this context, the value of the parameter $h$ has been obtained by minimizing the difference between the measured and the estimated torques, with a non-linear minimization criteria, on all the experimental acquisitions. The final value of $h$ is then computed as the mean value between the parameters estimated on all the trials. Secondly, in order to identify the friction temperature parameters, which are $\alpha, \beta, \gamma, \delta, K$ and $C$ for all the six joints, the same procedure described in [9] and [10] has been used. In particular, different duty cycles (DC) of motion (from 25\% to $100 \%$ ) have been performed and then, by using a minimization method based on the fmincon Matlab function, the estimation of the temperature parameters has been obtained.

The computed coefficients, for both inertial and friction models, have been validated with a different trajectory from the one used for the identification. As example of the validation results, the friction torque versus velocity curve of the second joint of the robot has been plotted. From Figure 3, we note that the friction torque obtained by subtracting from the motor torque of joint 2 the corresponding inertial torque (blue line) and the friction torque obtained from the proposed thermal model (red dashed line) are very similar. 

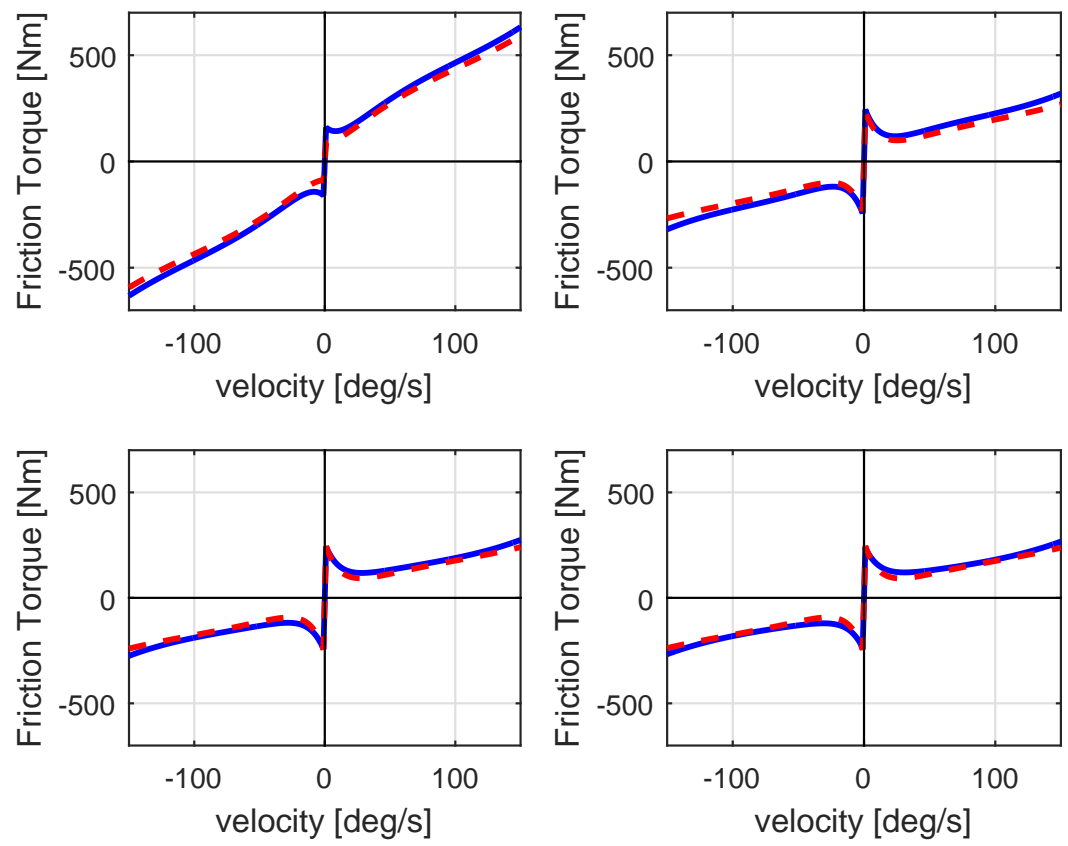

Fig. 3. Validation of the thermal friction model. Friction torque versus velocity plot for joint 2. Friction torque obtained with experimental data (blue) and identified with the proposed model (red). Top left: after 3 mins. Top right: after 78 mins. Bottom left: after 153 mins. Bottom right: after 228 mins.

\section{ViRTUAL FORCE SENSOR}

The virtual force sensor has been obtained by considering only the measurement of currents (i), positions $(\mathbf{Q})$ and velocities $(\dot{\mathbf{Q}})$ for all the six motors of the robot. The motors torques $(\tau)$ have been computed by considering the motor torque constants $\boldsymbol{K}_{\boldsymbol{t}}$ as

$$
\tau=K_{t} i
$$

and the accelerations $(\ddot{\mathbf{Q}})$ have been obtained by differentiating the motors velocities and by applying a low-pass filter.

The values of the external forces wrench $\mathbf{F}_{e}$ estimated by the virtual force sensor, for each time instant, have been computed by considering the following relation

$$
\mathbf{F}_{e}=\mathbf{J}(\mathbf{Q})^{-T} \boldsymbol{\delta} \tau
$$

where $\mathbf{J}(\mathbf{Q})$ is the robot Jacobian matrix and $\boldsymbol{\delta} \tau$ is the vector of the joints extra torques. These extra torques have been determined as

$$
\delta \tau=\tau_{o}-\tau_{f}
$$

where $\tau_{f}$ is the vector of the joints friction torques and $\tau_{o}$ represents the vector that is obtained as the difference between the complete motors torques $\tau$ and the torques related to the to masses and inertial components $\tau_{J}$

$$
\tau_{o}=\tau-\tau_{J}
$$

For each time interval, the torques related to masses and inertial components have been obtained as

$$
\tau_{J}=\boldsymbol{\Phi}_{J} \mathbf{X}_{J}
$$

where $\boldsymbol{\Phi}_{\boldsymbol{J}}$ represents the regressor matrix computed at the considered time instant and $\mathbf{X}_{J}$ is the vector of the estimated dynamic parameters.

\section{EXPERIMENTAL PROTOCOL}

Before presenting the experimental protocol of this work, it is necessary to highlight some issues. First of all, an observer based strategy has been adopted in order to allow the temperature observer to adjust the initial temperature for all the six joints of the robot (see (10)). It is worth stressing that such a strategy has been used only at the beginning of the process in order to find the initial conditions of the temperature. During the activation of the observer, it has to be ensured that there are no contacts between the robot and the environment. In fact, without any contact $\left(\tau_{e}=0\right)$, (18) represents only the friction torques, i.e.

$$
\tau_{o}=\tau_{f}
$$




\begin{tabular}{|c|c|c|}
\hline Cut-off freq [rad/s] & Data & Symbol \\
\hline 150 & Measured motor currents & $\mathbf{i}$ \\
\hline 150 & Computed accelerations & $\ddot{\mathbf{Q}}$ \\
\hline 150 & Inertial torques & $\boldsymbol{\tau}_{J}$ \\
\hline 150 & Friction torques & $\boldsymbol{\tau}_{f}$ \\
\hline 1 & Extra torques & $\boldsymbol{\tau}_{e}$ \\
\hline 15 & External forces & $\mathbf{F}_{e}$ \\
\hline
\end{tabular}

TABLE II

CUT-OFF FREQUENCIES FOR THE DIFFERENT LOW PASS FILTERS THAT HAVE BEEN APPLIED ON THE VIRTUAL FORCE SENSOR.

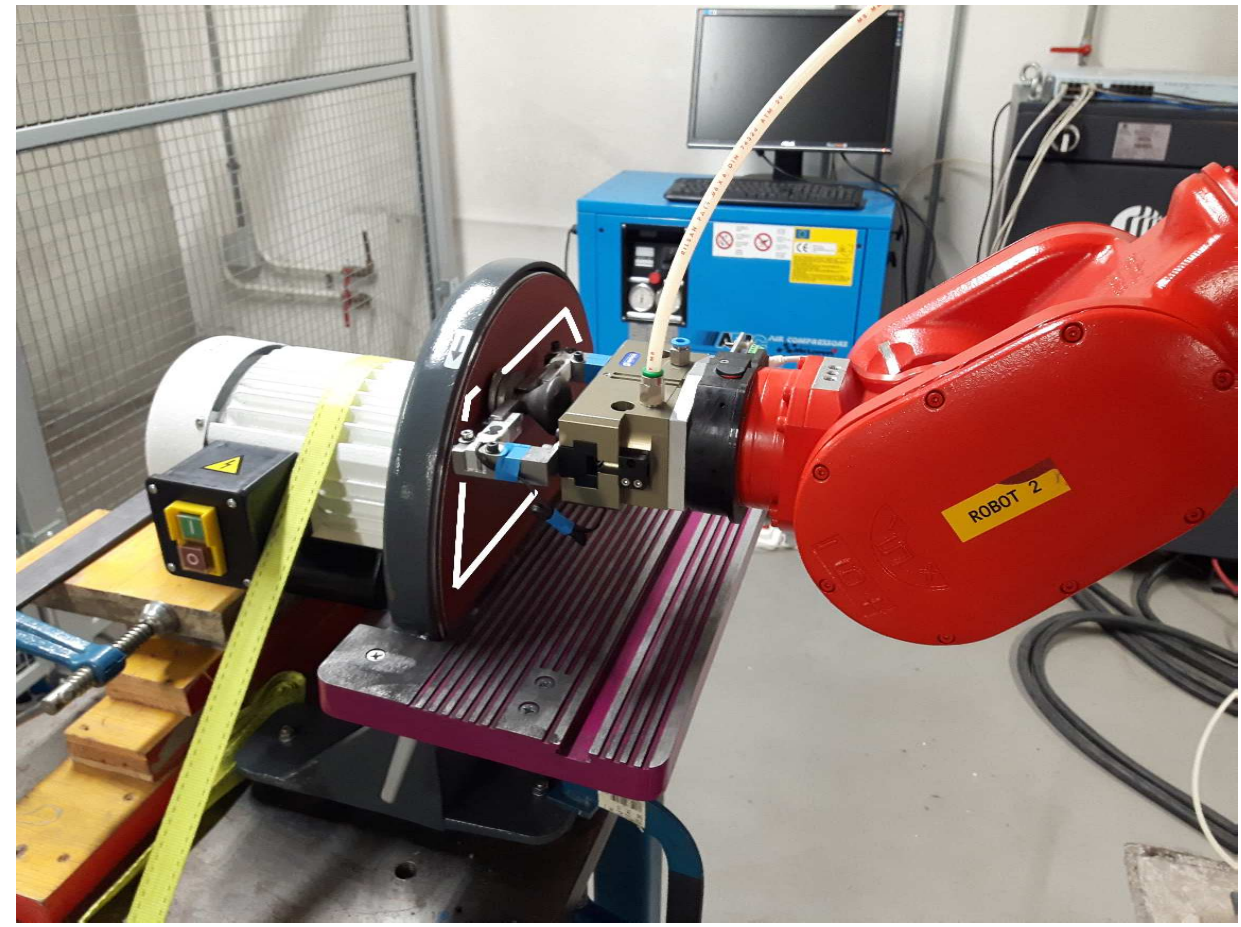

Fig. 4. End effector motion. The white path is the path of the end effector during the polishing application.

and therefore the initial temperature can be correctly estimated by using the measurements of the motor currents. A sampling period $t_{s}$ equal to $1.2[\mathrm{~ms}]$ has been used for the proposed virtual force sensor. The data have been low-pass filtered with the cut-off frequencies shown in Table II. These cut-off frequencies have been selected experimentally. Finally, as in real industrial polishing applications the disc sander has to work for several hours without being ruined and as the surface to polish has to be very flat, the contact region has to be changed during the cycle. For this reason, the iron sample has been moved along a predefined path on the disc sander surface. This path is composed by four points connected by four straight lines (see Figure 4). This motion have been performed to minimize the wear of the disc sander and to avoid the scratches on the sample surface.

The test protocol used in this work is:

1) at the beginning, the robot has been moved close to the disc sander, without touching the abrasive surface, for two minutes in order to allow the temperature observer to adjust the initial temperature for all the six joints of the robot;

2) the polishing task has been performed for two minutes (with the sample in contact with the disc sander surface);

3) the robot has been moved close to the disc sander, without touching the abrasive surface, for fifteen minutes;

4) point 2 and 3 of this list have been repeated six times.

During these experimental trials, a measurement of the contact force along the normal direction with respect to the disc sander surface has been performed by means of the load cell. It is worth stressing that these measurements have been done in order to compare the real contact force results with the ones estimated by the proposed virtual force sensor, but the load cell is not employed at all in the virtual sensing strategy.

\section{EXPERIMENTAL RESULTS}

The obtained results are shown in Figures 5-8 and summarised in Table III. The initial joint temperature estimation performed with the observer is shown in Figure 5 while the Figures 6-8 are related to the first, the third and the fifth cycles 


\begin{tabular}{|c|c|c|c|}
\hline & Contact cycle 1 & Contact cycle 3 & Contact cycle 5 \\
\hline with T & 21.3078 & 21.7498 & 21.2590 \\
\hline without T & 27.2322 & 27.4293 & 25.6366 \\
\hline
\end{tabular}

TABLE III

RMS ERROR OF THE ESTIMATED CONTACT FORCE.

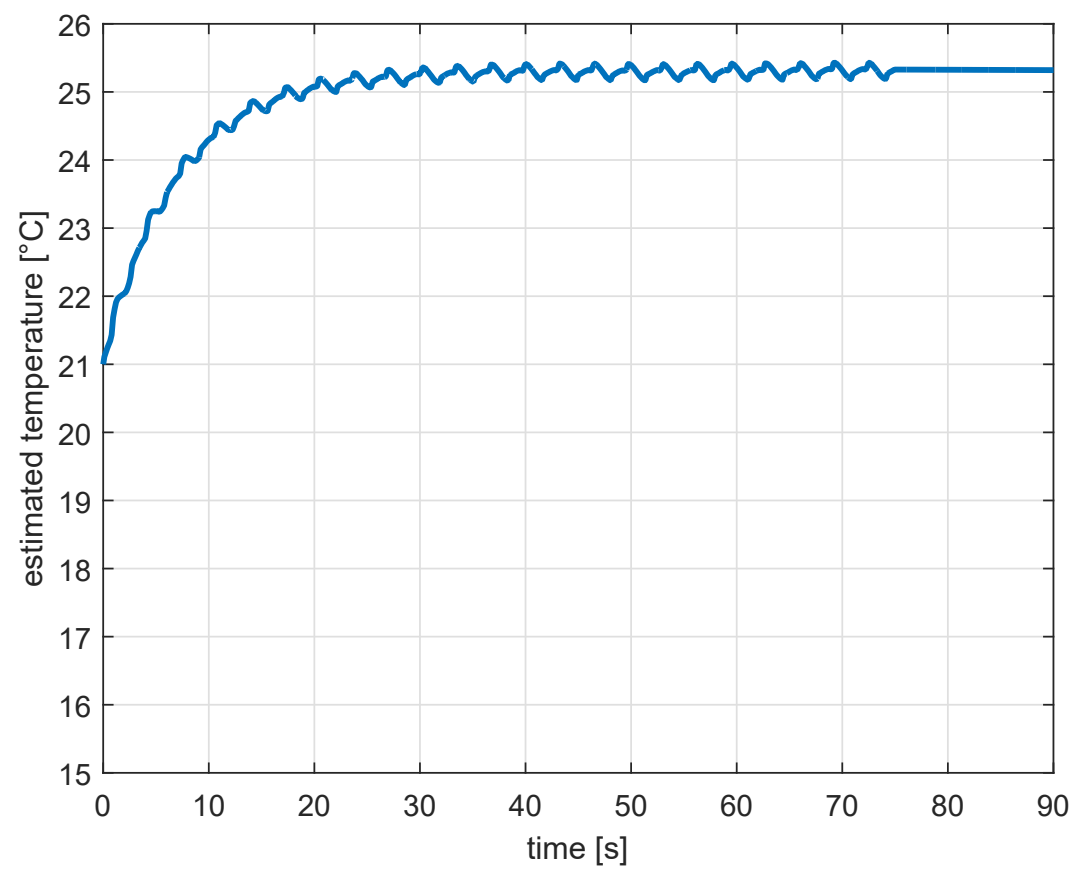

Fig. 5. Estimated temperature for joint 1 during the time interval in which the observer (10) is applied.

of the polishing work; that is, after $150 \mathrm{~s}$, after about $2240 \mathrm{~s}$ and after about $4320 \mathrm{~s}$ of robot operations, respectively (these time intervals have been selected by considering the thermal time constant of the robot joints). Figure 5 shows clearly the temperature observer output. Starting from a wrong initial temperature the observer is able to quickly compensate the temperature error. Thus, the initial time interval when the robot should not be in contact with the environment, can be relatively short. Figures 6-8 show, together, the contact force measured by using the load cell (green lines), the contact force estimated with the proposed virtual force sensor (blue dotted lines) and the one estimated with the virtual force sensor obtained without considering the joint internal temperature (red dashed lines). As it is possible to notice, the estimated force obtained from the virtual force sensor that considers the joint internal temperatures is better than the one obtained by using the virtual force sensor but without taking into account the joint temperatures; this consideration is confirmed by evaluating the RMS errors of the estimated contact force with end without considering the joint temperature (Table III). In fact the estimated contact force obtained with the proposed virtual force sensor is closer to the one measured with the load cell, than the estimated contact force obtained with the virtual force sensor obtained without using a temperature based friction model. Furthermore, from Figures 6-8 and Table III) it is possible to notice that: $(i)$ the error is greater when the joint internal temperature is not considered; ( $i i)$ the temperature dependency allows a better contact force estimation not only at the beginning of the polishing task, (that is just after the estimation of the initial joints temperatures), but also during the first hour of robot operations; It is in fact possible to see from these figures that the red dashed lines shift upwards from the first to the last contact trials, and it means that the temperature growth must be considered; (iii) the adopted friction model, that is the Stribeck plus polynomial one, does not represent perfectly the physics of the system when the sign of the speed of motion changes. Nevertheless the virtual force sensor in which the temperature dependency is considered is more precise than the other one; (iv) both the results of the two virtual force sensors have a small time delay related to the filtering process. It is worth stressing that the joint temperature is updated in open-loop by means of [9]. However, as the observer transient is very small (just a couple of minutes are necessary for the estimation) it is possible to re-estimate the temperatures (for example every thirty minutes), in order to avoid possible (small) drifts, by exploiting time intervals in which the robot is not in contact with the environment. 


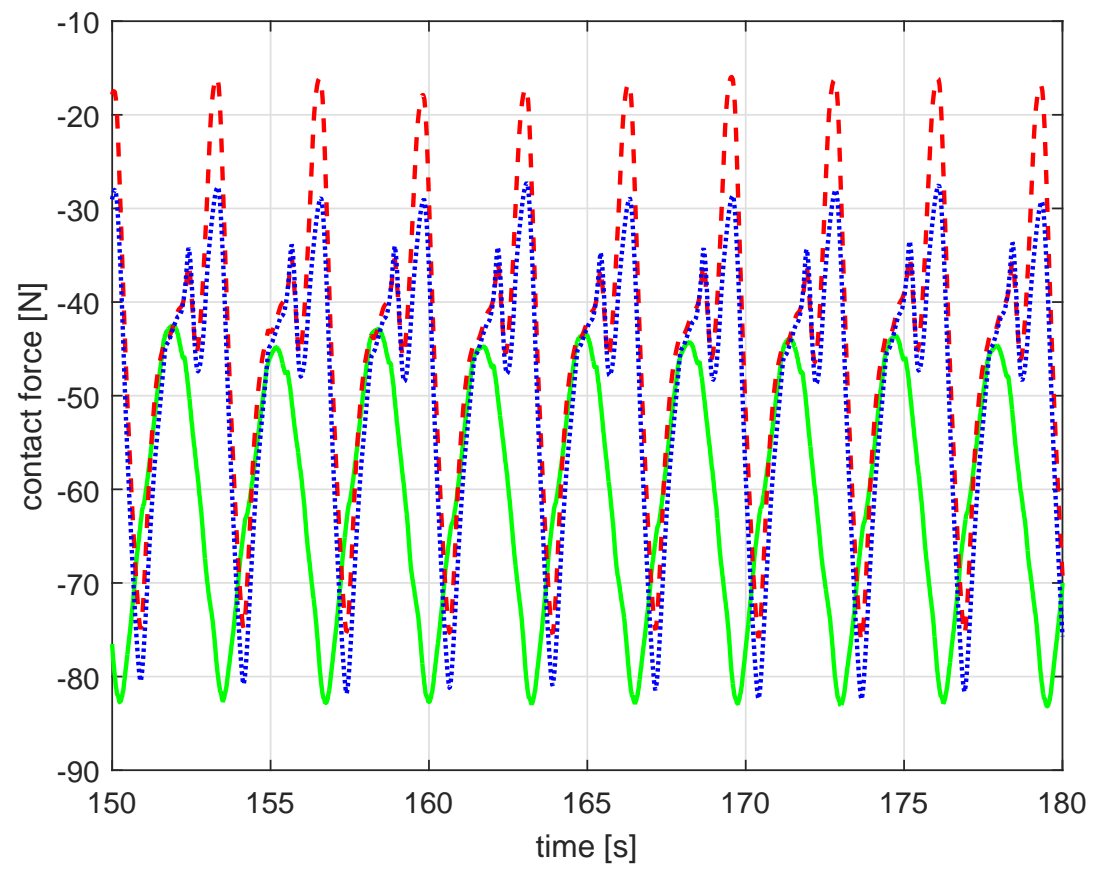

Fig. 6. Contact force measured by using the load cell (green line) compared with the one estimated by the proposed virtual force sensor (blue dotted line) and the one obtained with the virtual force sensor without considering the joint internal temperature (red dashed line).

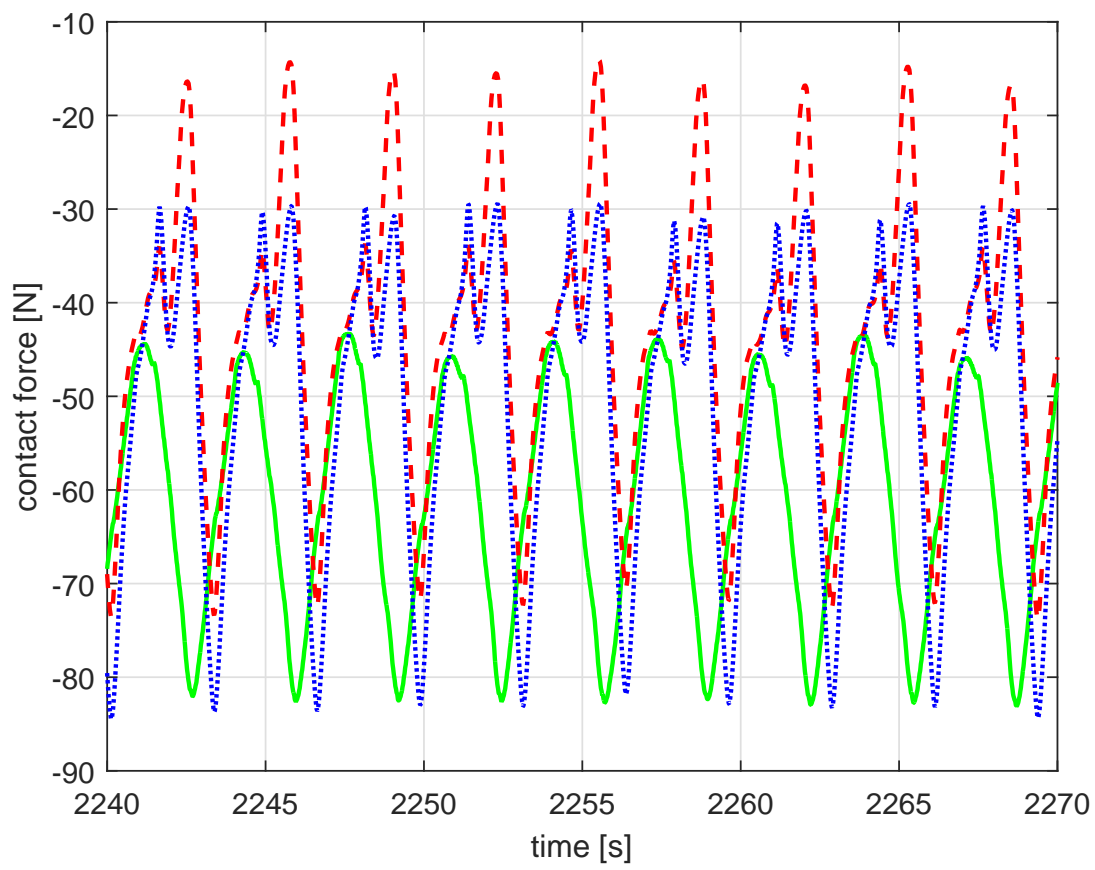

Fig. 7. Contact force measured by using the load cell (green line) compared with the one estimated by the proposed virtual force sensor (blue dotted line) and the one obtained with the virtual force sensor without considering the joint internal temperature (red dashed line). 


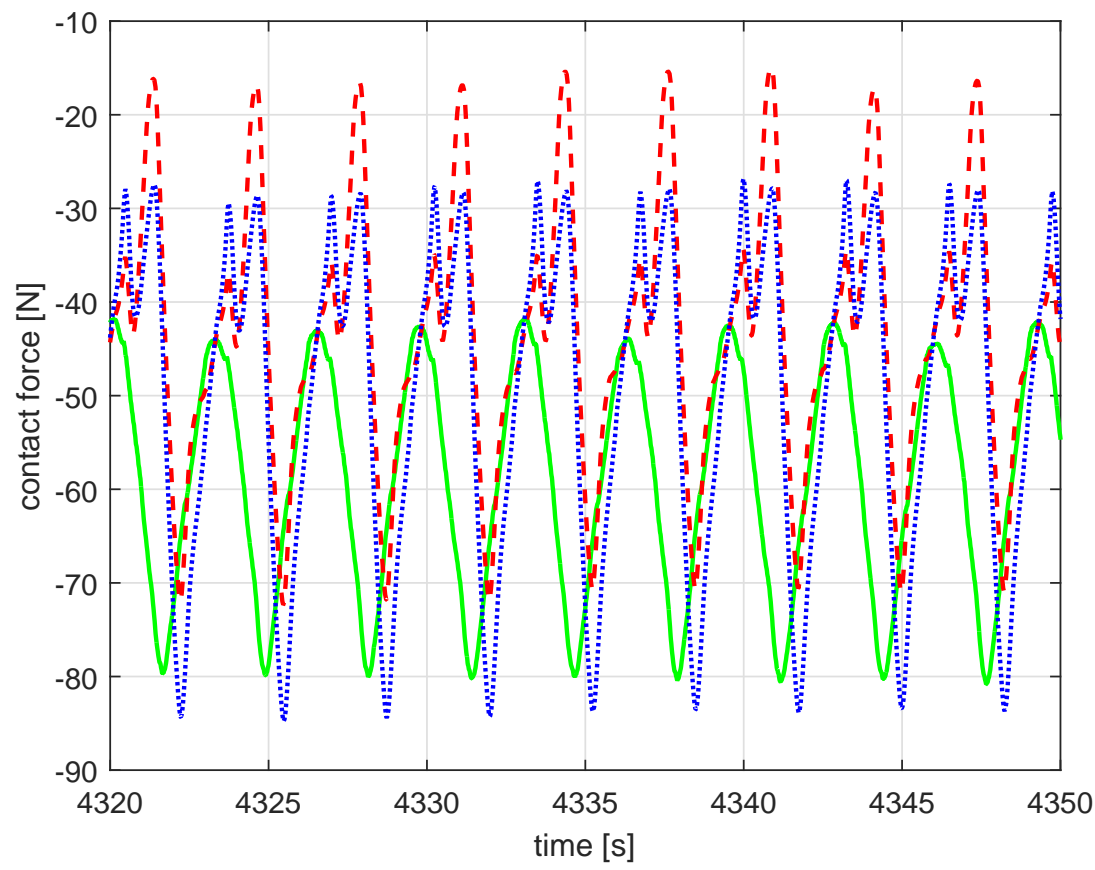

Fig. 8. Contact force measured by using the load cell (green line) compared with the one estimated by the proposed virtual force sensor (blue dotted line) and the one obtained with the virtual force sensor without considering the joint internal temperature (red dashed line).

\section{CONCLUSIONS}

In this paper we have investigated the use of a temperature based friction model to create a virtual force sensor for industrial robot manipulators. A comparison with a virtual force sensor that does not include a temperature based friction model has been done by evaluating the RMS of the estimated contact force. The experiments have shown that the dependency from the temperature of the friction model needs to be evaluated. In particular, an accurate joints temperature estimation enables to use the robot dynamic model for an accurate estimation of the forces exchanged between the robot and the environment.

Future work will consist in implementing a virtual force sensor built by considering a LuGre based thermal friction model [6] in order to reduce the estimated force error when the robot joints are close to zero velocities.

\section{ACKNOWLEDGMENTS}

The research leading to these results has received funding from the European Union H2020 program under grant agreement n. 637095 (FourByThree) and ECSEL-2016-1 under grant agreement n. 737453 (I-MECH)

\section{REFERENCES}

[1] M. Gautier and W. Khalil, "On the identification of the inertial parameters of robots," in Proceedings of the 27th IEEE/RSJ International Conference on Decision and Control (CDC), Austin (USA), 1988, pp. 2264-2269.

[2] K. R. Kozlowski, Modelling and Identification in Robotics. London (UK): Springer-Verlag, 1998.

[3] E. Berger, "Friction modeling for dynamic system simulation," Applied Mechanics Reviews, vol. 55, pp. 535-577, 2012.

[4] V. van Geffen, "A study of friction models and friction compensation," Technische Universiteit Eindhoven, Department Mechanical Engineering, Dynamics and Control Technology Group, Eindhoven (NL), Tech. Rep. DCT 2009.118, 2009.

[5] P. R. Dahl, "A solid friction model," Space and Missile Systems Organization, Air Force System Command, Los Angeles, CA (USA), Tech. Rep. ADA041920, 1968.

[6] K. J. Åström, C. C. de Wit, H. Olsson, and P. Lischinsky, "A new model for control of systems with friction," IEEE Transaction on Automatic Control, vol. 40, no. 3, pp. 419-425, 1995.

[7] K. J. Åström and C. C. de Wit, "Revisiting the lugre model," IEEE Control Systems Magazine, vol. 28, no. 6, pp. 101-114, 2008.

[8] V. Lampaert, F. Al-Bender, and J. Swevers, "A generalized maxwell-slip friction model appropriate for control purposes," in Proceedings of IEEE International Conference on Physics and Control, vol. 4, St. Petersburg (Russia), 2003, pp. 1170-1177.

[9] L. Simoni, M. Beschi, G. Legnani, and A. Visioli, "Friction modeling with temperature effects for industrial robot manipulators," in Proceedings of the IEEE/RSJ International Conference on Intelligent Robots and Systems, Hamburg (D), 2015, pp. 3524-3529.

[10] G. Legnani, L. Simoni, M. Beschi, and A. Visioli, "A new friction model for mechanical transmissions considering joint temperature," in Proceedings of the ASME 2016 International Design Engineering Technical Conferences and Computers and Information in Engineering Conference, Charlotte (US), 2016, pp. V006T09A020-V006T09A020.

[11] L. Márton and F. van der Linden, "Temperature dependent friction estimation: Application to lubricant health monitoring," Mechatronics, vol. 22, pp. 1078-1084, 2012.

[12] A. C. Bittencourt and S. Gunnarsson, "Static friction in a robot joint-modeling and identification of load and temperature effects," Journal of Dynamic Systems, Measurement, and Control, vol. 134, no. 5, 2012. 
[13] S. Pellegrinelli, S. Borgia, N. Pedrocchi, E. Villagrossi, G. Bianchi, and L. M. Tosatti, "Minimization of the energy consumption in motion planning for single-robot tasks," Procedia CIRP, vol. 29, pp. 354-359, 2015.

[14] E. Magrini, F. Flacco, and A. D. Luca, "Estimation of contact forces using a virtual force sensor," in Proceedings of the IEEE/RSJ International Conference on Intelligent Robots and System, Hamburg (D), 2014, pp. 2126-2133.

[15] A. D. Luca, A. Albu-Schaffer, S. Haddadin, and G. Hirzinger, "Collision detection and safe reaction with the dlr-iii lightweight manipulator arm," in Proceedings of the IEEE/RSJ International Conference on Intelligent Robots and Systems, Beijing (China), 2006, pp. 1623-1630.

[16] W. Chen, D. Ballance, P. Gawthrop, and J. OReilly, "A nonlinear disturbance observer for robotic manipulators," IEEE Transaction on Industrial Electronics, vol. 47, pp. 932-938, 2000.

[17] A. Nikoobin and R. Haghighi, "Lyapunov-based nonlinear disturbance observer for serial n-link robot manipulators," pp. 135-153, 2009.

[18] D. Pirro, M. Webster, and E. Daschner, Lubrication Fundamentals, Revised and Expanded. CRC Press, 2016.

[19] B. Siciliano and O. Khatib, Springer handbook of robotics. Springer, 2016.

[20] E. Villagrossi, N. Pedrocchi, F. Vicentini, and L. M. Tosatti, "Optimal robot dynamics local identification using genetic-based path planning in workspace subregions," in Proceedings of the IEEE/ASME International Conference on Advanced Intelligent Mechatronics, Wollongong (AUS), 2013, pp. $932-937$. 